\title{
Mycobacterium szulgai pulmonary infection in a vitamin D-deficient patient: A case report
}

\author{
Hadi Lotfi ${ }^{1}$, mojtaba sankian ${ }^{2}$, Zahra Meshkat $^{1}$, ahmad khalifeh soltani ${ }^{2}$, and ehsan $\operatorname{aryan}^{2}$ \\ ${ }^{1}$ Mashhad University of Medical Sciences \\ ${ }^{2}$ Affiliation not available
}

October 27, 2020

\begin{abstract}
We reported a case of M. szulgai respiratory infection in a 32-year-old male patient without any history of smoking, immunodeficiency syndromes, or receiving immunosuppressive medications but the patient was severely deficient in vitamin D. isoniazid, rifampin, ethambutol, clarithromycin antimicrobial agents with vitamin B6 and vitamin D.improved after 10 months.

Mycobacterium szulgai pulmonary infection in a vitamin D-deficient patient: A case report

Authors: Hadi Lotfi ${ }^{1}$, Mojtaba Sankian ${ }^{2}$, Zahra Meshkat ${ }^{1,3}$, Ahmad Khalifeh Soltani ${ }^{4}$ and Ehsan Aryan ${ }^{1,3,}$ *.

${ }^{1}$ Antimicrobial Resistance Research Center, Mashhad University of Medical Sciences, Mashhad, Iran

${ }^{2}$ Immunobiochemistry Laboratory, Immunology Research Center, Mashhad University of Medical Sciences, Mashhad, Iran

${ }^{3}$ Department of Medical Microbiology, Ghaem University Hospital, Mashhad University of

Medical Sciences, Mashhad, Iran

${ }^{4}$ Department of Infectious Diseases and Tropical Medicine, Faculty of Medicine, Mashhad University of Medical Sciences, Mashhad, Iran

${ }^{*}$ Corresponding author. Ehsan Aryan; postal address: Laboratory of Microbiology, Department of Medical Microbiology, Ghaem University Hospital, Mashhad University of Medical Sciences, Ahmad Abad Street, 91967-73117 Mashhad, Iran.Tel.: +98 511 38453019; fax: +98 5117112596.
\end{abstract}

E-mail address: ehsanaryan@hotmail.com

Institutions: Mashhad University of Medical Sciences

Authorship: The authors contributed equally to all aspects of the article.

Declaration of Conflicting Interest : The Authors declare that there is no conflict of interest.

Funding : The authors disclosed receipt of the following financial support for the research resulted in this report [grant number 961176].

Ethical Approval (include full name of committee approving the research and if available mention reference number of that approval): Ethical approval to report this case was obtained from MASHHAD UNIVERSITY of MEDICAL SCIENCES REGIONAL ETHICS COMMITTEE, Mashhad University of Medical Sciences, Mashhad, Iran (APPROVAL NUMBER: IR.MUMS.fm.REC.1396.638). 


\begin{abstract}
Mycobacterium szulgai (M. szulgai) is an opportunistic and rare pathogen. Several risk factors play roles in the development of disease caused by this bacterium including immunodeficiency syndromes, previous Mycobacterium tuberculosis infection and smoking. We reported a case of M. szulgai respiratory infection in a 32-year-old male patient without any history of smoking, immunodeficiency syndromes or receiving immunosuppressive medications but the patient was severely deficient in vitamin D. Finally, the following treatment regimen was administered to the patient: isoniazid, rifampin, ethambutol, clarithromycin antimicrobial agents supplemented with vitamin B6 and vitamin D. The patient's clinical condition was improved after 10 months of treatment. Vitamin D deficiency should be considered as a risk factor for M. szulgai pulmonary infection in immunocompetent people who are daily exposed to very little sunlight due to weather, occupational, or religious reasons.
\end{abstract}

Key Clinical Message: Closer attention should be paid to vitamin D status in patients with mycobacterial diseases.

Keywords : Mycobacterium szulgai, pulmonary infection, vitamin D deficiency, case report

\title{
Background
}

Nontuberculous mycobacteria (NTM) are widespread worldwide. These bacteria can be transmitted to humans from environmental sources such as soil and water with no reported cases of person-to-person transmission [1].

Mycobacterium szulgai (M. szulgai ) is a slow-growing NTM and a rare human pathogen, representing $<0.2 \%$ of all NTM infections. [2]. M. szulgai pulmonary infections are infrequently reported in those with a history of chronic obstructive pulmonary disease (COPD), tuberculosis, or immunosuppression [3]. Clinically, it is indistinguishable from Mycobacterium tuberculosis infection and neoplasm, therefore, it is critical to be diagnosed accurately [4]. Moreover, there is no consensus recommendation for treatment of M. szulgai infections, probably due to its low prevalence [5]. Herein, we present a case of M. szulgai pulmonary infection in an immunocompetent patient without any history of tuberculosis or consumption of immunosuppressive drugs but severely deficient in vitamin D that was successfully treated in Mashhad, Iran.

\section{Case presentation}

A 32-year-old man was referred to Ghaem Hospital affiliated to Mashhad University of Medical Sciences in February 2019 with symptoms of night sweats, cough, and chest pain with previous pulmonary infectionmycobacterium tuberculosis diagnosis based on positive acid-fast bacilli smear test. The patient was received a standard 6-month regimen including rifampin, isoniazid, ethambutol and pyrazinamide. He had no history of alcohol use and smoking. The patient had a weight loss of $9 \mathrm{~kg}$ over one year. The patient worked in a clothing industry that was not exposed to sunlight. An initial test performed on the patient showed severe vitamin D deficiency. A serology test for HIV was negative. The chest X-ray revealed an increase in pleural thickness was seen in the left lung and less strongly in the right lung. Reticular lesions were observed in the field of lungs, specifically, at the top of the lungs (Figure 1A). High-resolution computed tomography (HRCT) revealed bronchiectasis, distractive changes and multiple cysts with collapse in the left upper lobe. Bronchiectasis and cyst formation with tree-in-bud pattern were observed in the right upper lobe and less intensively in the right middle lobe and upper left lower lobe segment and with less intensity in the upper right lobe (Figure 1C). Laboratory findings (table 1) combined with the clinical signs of the patient raised the possibility of Mycobacterium tuberculosis infection (TB). Therefore, direct smear microscopy for acid-fast bacilli (AFB) and mycobacterial culture were performed on the patient's sputum sample. All three samples obtained from the patient were smear-positive for AFB using Ziehl-Neelsen method. Also, mycobacterial culture of the patient's samples on Lowenstein-Jensen medium became positive for AFB twenty-two days after inoculation. To identify the grown bacterium, two independent PCR assays were performed on the DNA extracted from the bacterial colonies. One PCR assay had been designed to detect IS6110, a specific gene target for $M$. tuberculosiscomplex, in the specimen and the other assay detected rpo $B$, a mycobacterium 
genus-specific target. The organism was identified as (NTM) due to obtaining negative and positive results respectively for the former and the latter PCR assays. results were also confirmed by sequencing of the ITS (16S-23S) (accession number MN124505), rpoB (accession number MN158122), and hsp65 (accession number MN158123) genes of M. szulgai . Therefore, treatment with isoniazid (225mg/daily), rifampicin (450mg/daily), ethambutol (825mg/daily), clarithromycin $(1000 \mathrm{mg} /$ daily), vitamin B6 (10 mg/day) and vitamin D (1000 IU/day) was started. After ten months of treatment, a sputum sample was taken from the patient, smear, and culture tests were negative. Chest pain and dyspnea were resolved and the patient gained $6 \mathrm{~kg}$. Significant improvement was seen in the chest X-ray (Figure 1B). The patient's serum vitamin D also reached normal.

\section{DISCUSSION}

According to the ATS (American thoracic society) and IDSA (infectious disease society of America) guidelines released in 2007, to identify NTM respiratory infection radiological, microbiological, and clinical findings are required. These include finding nodular or cavitary lesions on chest X-ray or bronchiectasis on HRCT and positive culture of at least two sputum specimens[6]. Most cases of M. szulgai respiratory infection have been previously reported to be fibrocavitary[5]. whereas in our case was bronchiectasis with nodular lesions and a tree-in-bud view. In the bronchiectasis form, the disease is more scattered in the middle lobe and lingual area, whereas in our case most lesions were in the upper lobe. Lio et al. showed that the bronchiectasis form of Mycobacterium avium pulmonary infection is more commonly seen in those with chronic obstructive pulmonary disease or gastrointestinal malignancies such as gastroesophageal reflux disease[7]. Our patient had no history of any obstructive diseases or gastrointestinal malfunctions. In the majority of reported cases, M. szulgai respiratory infections men over 50 years of age and have risk factors such as alcohol abuse, smoking, history of TB infection, low body mass index or skeletal abnormalities of the chest[8]. To our knowledge, the present patient is the youngest case of $M$. szulgai pulmonary infection without any underling disease with had severe vitamin $\mathrm{D}$ deficiency. Vitamins can affect different components of the innate immune system, and deficiencies lead to defects in the immune system and infections[9]. The normal blood level of vitamin $\mathrm{D}$ is about $30 \mathrm{ng} / \mathrm{ml}$ and vitamin D deficiency involves a blood level below $20 \mathrm{ng} / \mathrm{ml}$. Douglas et al. demonstrated that vitamin D plays an important role in immunological defense against mycobacterial infections. Vitamin D activates macrophages by blocking the intera cellular growth of mycobacteria; on the other hand, vitamin D deficiency causes immune system defeat and growth Mycobacteria easily [11]. The main source of vitamin D for humans is sunlight [12]. Because the patient spent a lot of time at the workplace and no history of eating vitamin D supplements and Vitamin D rich diets, he had a severe vitamin D deficiency.

Unlike other NTM, M. szulgai is sensitive to most anti-TB drugs and responds well to treatment regimens containing more than two anti-TB drugs [6]. M. szulgai is also sensitive to macrolides aminoglycosides, and fluoroquinolones. In some reports, clarithromycin treatment with ethambutol and rifampicin or rifabutin has been found effective [7]. Clarithromycin is one of the famous members of aminoglycosides which has been FDA approved in 1990. This therapeutic agent is recommending for various bacterial infection particularly mycobacterial diseases [10]. According to review of the literatures, there are several evidence for successful treatment of clarithromycin in pulmonary infection caused by M. szulgai [11]. On a 10-month follow-up, the patient had a negative smear and culture. Signs of improvement in the patient's chest X-ray visible (Fig. B).

\section{CONCLUSION}

M. szulgai can infect immunocompetent individuals with risk factors such as vitamin $\mathrm{D}$ deficiency. The positive effect of vitamin D Supplement in innate Immunity in response to mycobacterial Infection through the regulation of Macrophage Activity and Uses it's as a treatment for NTM. Closer attention should be paid to vitamin D status in patients with mycobacterial diseases.

\section{Reference}

1. Gido, R.D.S., A.L. Wojciechowski, and R.P. Bajwa, Pulmonary infection with Mycobacterium szulgai: A 
case report. SAGE open medical case reports, 2019. 7 : p. $2050313 X 18823448$.

2. Martín-Casabona, N., et al., Non-tuberculous mycobacteria: patterns of isolation. A multi-country retrospective survey. The International Journal of Tuberculosis and Lung Disease, 2004.8 (10): p. 1186-1193.

3. Marks, J., P. Jenkins, and M. Tsukamura, Mycobacterium szulgai-a new pathogen. Tubercle, 1972.53 (3): p. 210-214.

4. Khan, S., et al., Pulmonary Mycobacterium szulgai infection: a case report. QJM: An International Journal of Medicine, 2016.109 (7): p. 489-490.

5. Sánchez-Alarcos, J.M., et al., Pulmonary infection due to Mycobacterium szulgai. Respiration, 2003.70 (5): p. 533-536.

6. Griffith, D.E., et al., An official ATS/IDSA statement: diagnosis, treatment, and prevention of nontuberculous mycobacterial diseases. American journal of respiratory and critical care medicine, 2007.175 (4): p. $367-416$.

7. Lio, J., Y. Lee, and R. Obata, Comparison of Characteristics of Fibrocavitary and Bronchiectasis Phenotypes of Pulmonary Mycobacterium Avium, in A106. NTM: BENCH TO BEDSIDE . 2019, American Thoracic Society. p. A2524-A2524.

8. Lake, M.A., et al., "Why me, why now?" Using clinical immunology and epidemiology to explain who gets nontuberculous mycobacterial infection. BMC medicine, 2016.14 (1): p. 54.

9. Erickson, K.L., E.A. Medina, and N.E. Hubbard, Micronutrients and innate immunity. The Journal of infectious diseases, 2000.182 (Supplement_1): p. S5-S10.

10. Fischer, J., et al., Analogue-based drug discovery . 2010: Wiley-VCH Hoboken, NJ.

11. Basille, D., V. Jounieaux, and C. Andréjak. Treatment of other nontuberculous mycobacteria . in Seminars in respiratory and critical care medicine. 2018. Thieme Medical Publishers. 

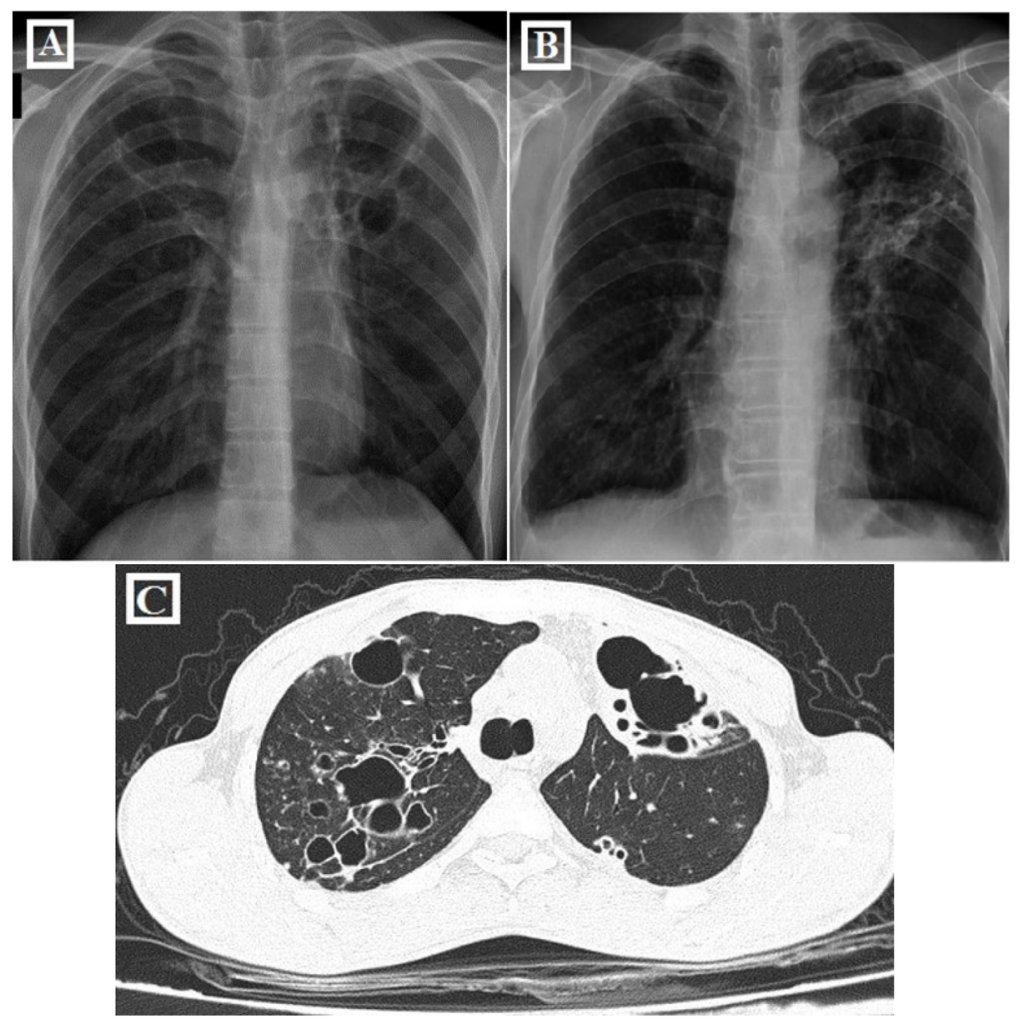

Figure 1: 32-year-old man with $M$. szulgai respiratory infection

A: Fibrotic changes in the lungs with preference to the left lung. B: Follow-up chest radiograph 10 months after treatment.C: Bronchiectasis and cyst formation with a tree-in-bud pattern in the right upper lobe and less strongly in the right middle lobe and upper left lower segments 